\title{
Pulse-mode $x$-ray beam position monitor prototype for a synchrotron radiation beam line
}

\author{
Hideki Aoyagi, Yukito Furukawa, Sunao Takahashi®, and Atsuo Watanabe \\ Japan Synchrotron Radiation Research Institute (JASRI), 1-1-1 Kouto, Sayo, Hyogo 679-5198, Japan
}

(Received 5 August 2020; accepted 22 February 2021; published 15 March 2021)

\begin{abstract}
An x-ray beam position monitor (XBPM) prototype that can be operated in pulse mode has been designed and demonstrated. The monitor has a microstripline structure for signal transmission lines and a titanium electrode sputtered on a diamond heat sink as a photocathode. The detection elements of this monitor generate unipolar single pulses with a full width at half maximum of less than $1 \mathrm{~ns}$, allowing a pulse-by-pulse measurement of the synchrotron radiation beam. By synthesizing these pulses, a directly readable position sensitive signal is provided from pulse to pulse. This monitor can be used in the direct current mode in which it exhibits a stability and resolution as good as those of conventional XBPMs. Results of the performance tests of the monitor at the SPring- 8 synchrotron radiation beam line are also presented.
\end{abstract}

DOI: 10.1103/PhysRevAccelBeams.24.032803

\section{INTRODUCTION}

X-ray beam position monitors (XBPMs) are widely used tools to provide information on the photon beam position and pointing stability in the front end in a synchrotron radiation facility. The standard XBPMs are photoemission type, and blade-shaped tungsten or diamond is used as the detection element to improve heat resistance [1-6]. In the SPring-8 storage ring, XBPMs are installed at the front end of all insertion device beam lines (ID-BLs) and most of the bending magnet beam lines (BM-BLs). The XBPM for an ID-BL has four detection elements arranged horizontally and vertically near the optical axis to measure the photon beam positions in the horizontal and vertical directions, respectively; for a BM-BL, the XBPM has two detection elements arranged vertically to measure the photon beam position in the vertical direction.

In synchrotron light sources, the number of timeresolved experiments, utilizing the pulse property of a synchrotron radiation (SR) beam to analyze the phenomena that develops in an extremely short time, is increasing [7-9]. For example, in a user experiment, time-resolved measurements of the electronic properties via x-ray pulse excitation are carried out by synchronizing the SR pulse with a wideband pulse laser by using pump-probe techniques. To perform these experiments stably and efficiently, the beam position fluctuations for each SR pulse must be

Published by the American Physical Society under the terms of the Creative Commons Attribution 4.0 International license. Further distribution of this work must maintain attribution to the author(s) and the published article's title, journal citation, and DOI. suppressed to at least $10 \%$ of the beam size. As the minimum spread of the first harmonic, generated in the undulator radiation, is approximately $10 \mu \mathrm{rad}$ root mean square (rms), the required resolution of the XBPMs, which are located at a distance of approximately $20 \mathrm{~m}$ from the undulators, is $20 \mu \mathrm{m}$. Even if the pulse intensity changes due to the difference in filling pattern of the storage ring, it is necessary to satisfy this condition. In addition, the short- and long-term beam positional stabilities are required to be of the order of $1 \mu \mathrm{m}$, as in the case of a conventional XBPM.

In the SPring-8, after extensive efforts, a high level of stabilization of the electron beam has been realized not only for short- and long-term periods but also for pulse-by-pulse stabilization [10-17]. It is crucial to verify pulse-by-pulse stabilities by directly observing the SR beam. However, the conventional XBPMs have a large stray electric capacitance owing to the physical size of the detection elements. The stray capacitance $(\mathrm{C})$ of tungsten blades of conventional XBPMs is typically $480 \mathrm{pF}$. This corresponds to a time constant $(t=C \cdot R)$ of $24 \mathrm{~ns}$. In addition, the blades in a vacuum chamber and feedthrough terminals are connected by bare wires, causing intense reflection. An RG-58 coaxial cable is used to transmit the signal from in the machine tunnel; therefore, attenuation also occurs in the highfrequency range. Thus, the time resolution of these conventional XBPMs is limited and it is not possible to measure each pulse of the SR beam. To solve this problem, a pulseby-pulse SR beam monitor with a microstripline structurer had been developed $[18,19]$. In this monitor, an impedance matching microstripline itself is used as the photocathode of the detection elements for optimum improvement of the high-frequency characteristics. It has already been demonstrated that the dynamics of the SR beam (intensity, 
position, and timing) can be observed pulse by pulse in the BM-BLs using this monitor [20]. However, to use this monitor in the ID-BLs, which have a significantly powerful SR beam, further improvements in the heat resistance property of the monitor are required.

Forming impedance-matched electrodes on the surface of a diamond heat sink by directly adopting the idea of a microstripline structure is considerably difficult, because it is not practically compatible with securing a cooling path to improve the heat resistance. Therefore, we accepted the impedance mismatch in the vicinity of the detection elements and tried to limit the increase in the time constant by optimally reducing the stray capacitance while securing an effective area of the photocathode [21].

\section{DESIGN STUDY}

We have considered the following points while proceeding with the design study of the proposed pulse-mode XBPM. Instead of directly irradiating the SR beam on the metal line of the microstripline structure, metal (titanium) is plated on the diamond heat sink as a photocathode. Four detection elements [upper-left (UL), upper-right (UR), lower-left (LL), and lower-right (LR)] are arranged almost parallel to the SR beam axis to reduce the effective irradiation cross section, as shown in Fig. 1. The diamond heat sink holder is mounted on a cooling base welded to an ICF70 flange, which is introduced from both top and bottom side ports. Separately, the signal transmission line with the microstripline structure, which is attached to the ICF70 flange [22], is introduced from both left and right side ports. By separating the cooling mechanism and the signal transmission line, the high-frequency characteristics are partially sacrificed compared to the signal transmission line with the microstripline structure, but the cooling efficiency is expected to be significantly improved.

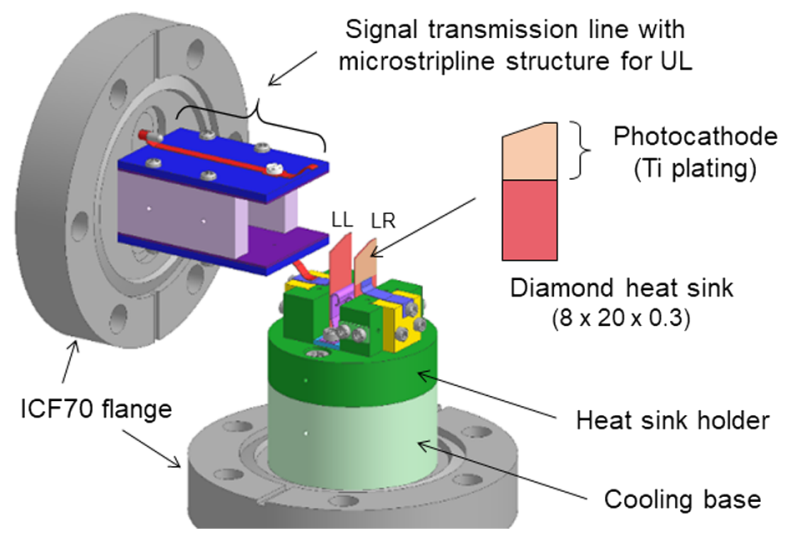

FIG. 1. Diamond heat sink (upper right) and configuration of transmission lines having microstripline structure and heat sinks mounted on a cooling base. In this figure, two lower heat sinks (LL, LR) out of four heat sinks and signal transmission lines for the two left-sided heat sinks (UL, LL) are shown.
Figure 2 shows a six-way cross chamber with a couple of detector holders mounted on the top and bottom ports and a couple of signal transmission lines mounted on the left and right ports.

\section{A. High-frequency characteristics}

To evaluate the high-frequency characteristics of the newly designed monitor, it is ideal to use a single $\mathrm{x}$-ray pulse (typical pulse length of $30 \mathrm{ps}$ rms) in the actual SR beam line. However, off-line tests, such as the time domain transmissiometry (TDT) and time domain reflectometry (TDR), can easily locate where the impedance mismatch occurs between the detector and the feedthrough terminal of the vacuum chamber. If a problem is found, then it can be corrected in advance. Therefore, TDT and TDR were carried out to evaluate the high-frequency characteristics of this monitor. Usually, a rectangular wave is used as a probe signal, and the variation at the rising or falling edges are analyzed. In our experiment, a single unipolar pulse [full width at half maximum (FWHM) $=140 \mathrm{ps}$ ] was used as the incident signal because the corresponding response signal was easy to interpret.

The actual usage of this monitor is to transmit the charge generated on the detection element at the tip of the diamond heat sink to a measuring instrument. Therefore, the time structure of an actual output signal can be predicted by observing the transmission of the unipolar signal, which is the input from the detection element via the probe. Figure 3 shows the setup of the TDT experiment. The input pulse from the pulse generator is divided into an oscilloscope trigger signal and a probe signal by the resistive power divider (HUBER + SUHNER 4901.19A). This method simulates a signal transmission path for a signal generated in the detection element. In this experiment, the diamond heat sink was replaced by a dummy to prevent handling failures. To obtain a response signal with less noise, it is

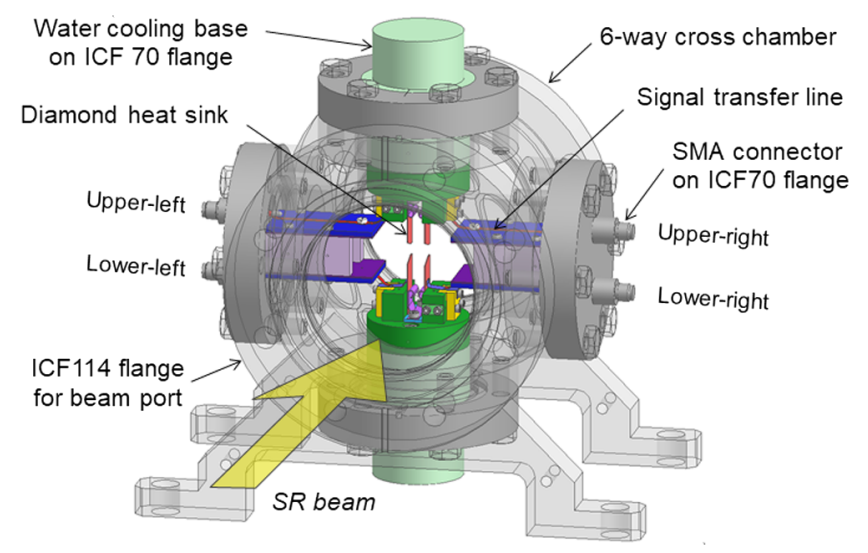

FIG. 2. Diagram of the six-way cross chamber with a couple of detector holders mounted on the top and bottom ports and a couple of signal transmission lines mounted on the left and right ports. 


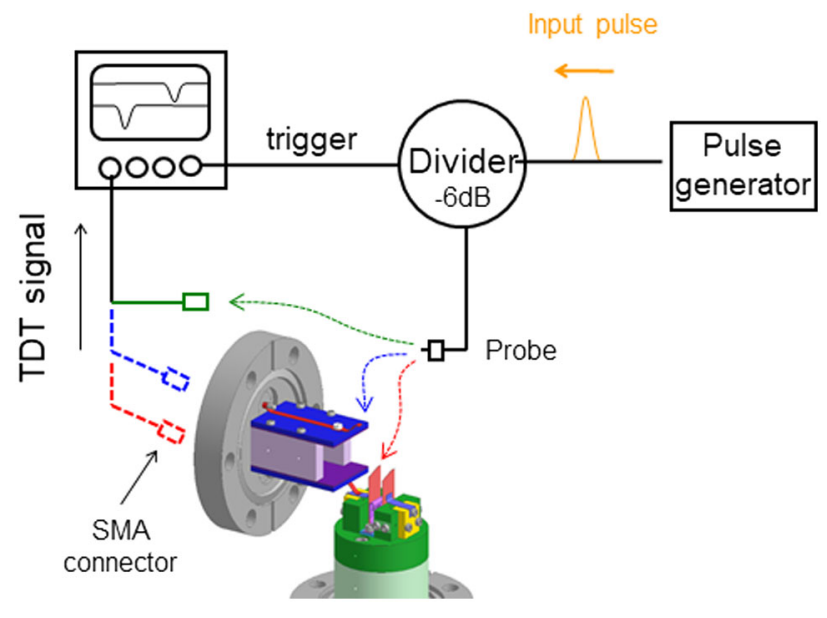

FIG. 3. Experimental setup of the TDT.

important to ground the shield wire of the probe to the vacuum chamber at a position near the core wire of the probe (not shown in the figure). Figure 4 shows the time structure of TDT signals in the following cases: (T-0) the trigger signal branched by the resistive power divider, (T-1) the direct signal when the cable of the probe is shortcircuited, (T-2) the transmitted signal when the probe is connected to the tip of the microstripline structure (the the SubMiniature version A (SMA) connector is connected to the upper feedthrough of ICF70), and (T-3) the transmission signal when the probe is connected to the tip of the detection element (the SMA connector is connected to the lower feedthrough of ICF70). Although the output signal from the tip of the microstripline structure (T-2) has a $0.4 \mathrm{~ns}$ delay (which corresponds to the time length of the microstripline structure) compared to the short-circuit signal (T$1)$, the time structure is largely retained. A double peak appears in the signal from the detection element (T-3) because of the impedance mismatch between the detection element on the diamond heat sink and the microstripline structure.

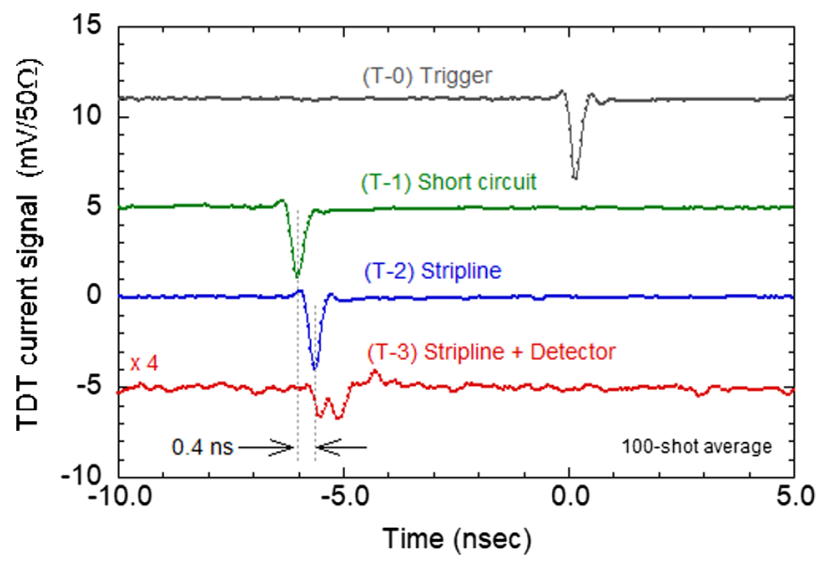

FIG. 4. Time structure of TDT signals. The signal of T-3, magnified 4 times, is displayed along the vertical axis.

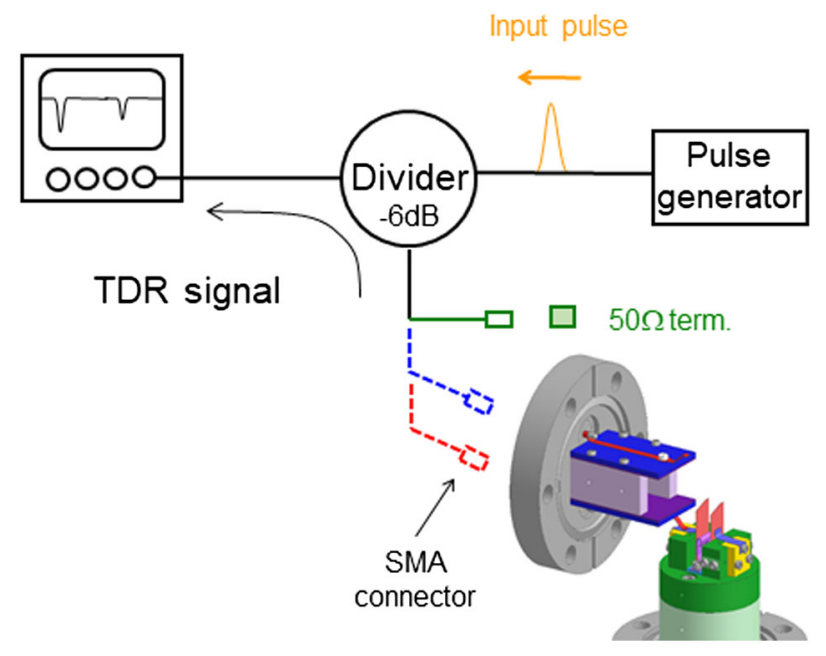

FIG. 5. Experimental setup of TDR.

Figure 5 shows the TDR setup. The input pulse from the pulse generator is branched into the oscilloscope and to the test object, and the TDR signal is received by the oscilloscope. TDR measurement is useful because in this method, the detector is protected by avoiding a direct contact between the probe and the detection element in the vacuum chamber. Figure 6 illustrates the measurement results of the TDR. When a $50 \Omega$ terminator is attached to the SMA connector, only the input pulse is observed (R-1). When the terminator is removed (open end), a reflected pulse is observed after a time delay of approximately $8.6 \mathrm{~ns}$, which is equivalent to twice the cable length (R-0). When the SMA connector is connected to the upper feedthrough of the ICF70, the TDR signal is observed with a delay of approximately $0.7 \mathrm{~ns}(\mathrm{R}-2)$. When it was connected to the lower feedthrough of the ICF70, the TDR signal shows a double-peak structure (R-3).

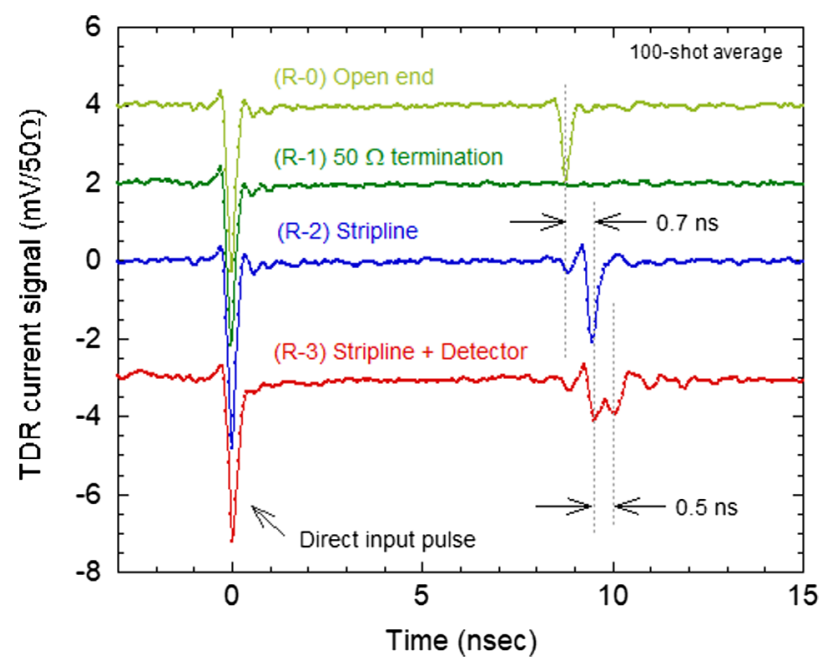

FIG. 6. Time structure of TDR signals. 


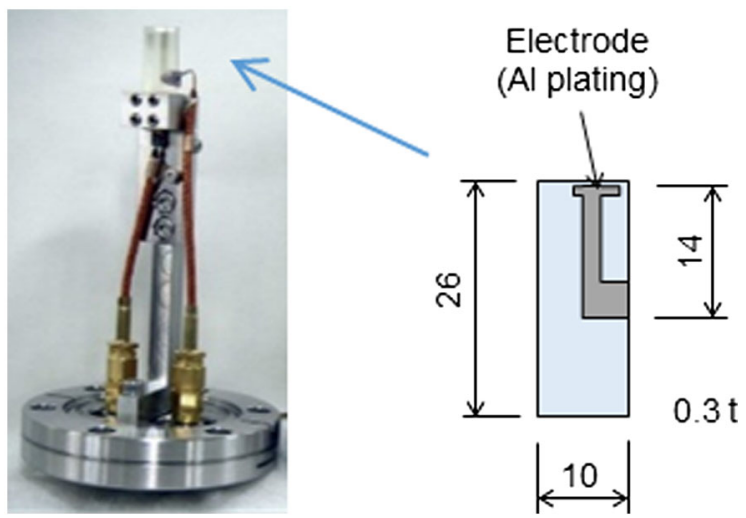

FIG. 7. Schematic of the detection element (right) and entire structure of the detection element including the ICF70 flange (left) developed as an electron beam halo monitor.

To clarify the relationship between the result obtained by TDR and the pulse waveform actually generated from the monitor, the same measurement was performed with a diamond semiconductor detector developed as an electron beam halo monitor [23]. Figure 7 shows a schematic of the detection element (right) and the actual structure of the entire detection element including the ICF70 flange (left). Figure 8 illustrates the TDR signal of the diamond semiconductor detector (R-4) and the signal generated by the injection of an electron pulse beam $(t=50 \mathrm{ps}$ FWHM) during an actual operation (R-5). The separation between the peaks in the double peak observed in TDR is approximately $0.5 \mathrm{~ns}$, whereas the FWHM of the actual output pulse was $0.4 \mathrm{~ns}$. In analogy to the diamond-based detector of the electron beam halo monitor, the TDR result of the pulse-mode XBPM suggests that unipolar single pulse signals with subnanosecond pulse length can be expected.

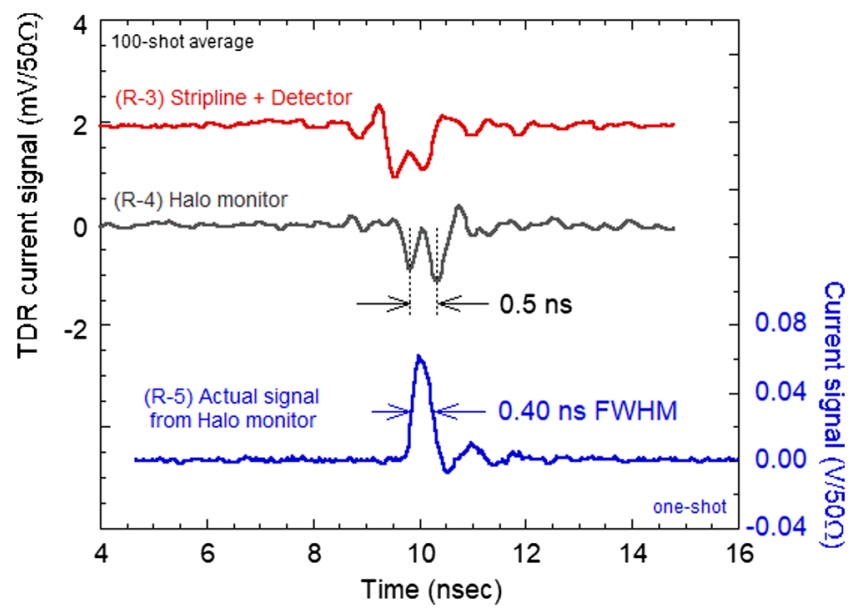

FIG. 8. Time structure of TDR signals and actual signal of electron beam halo monitor.

\section{B. Thermal finite element analysis}

As the SR beam intensity of ID-BLs is higher than that of BM-BLs, the heat-resistant design of detection elements for ID-BLs becomes extremely challenging. The beam powers and shapes of typical SPring-8 SR beam lines are as follows. In the BM-BLs, the beam power of SR beam has a uniform distribution in the horizontal direction and spreads in the vertical direction to approximately $1 / \gamma=$ $64 \mu \mathrm{rad}$ half width at half maximum (HWHM). Here, the Lorentz factor $(\gamma)$ is approximately 16,000 for SPring-8, where the energy of the electron beam is $8 \mathrm{GeV}$. The maximum power density is $1.5 \mathrm{~kW} / \mathrm{mrad}^{2}$. At a typical distance of about $12 \mathrm{~m}$ from the light source (BM) to the XBPM, the beam size is $0.77 \mathrm{~mm}$ HWHM in the vertical direction and the maximum power density is $10 \mathrm{~W} / \mathrm{mm}^{2}$. Contrarily, in the ID-BLs the beam power spreads in approximately 100(40) $\mu \mathrm{rad}$ HWHM in the horizontal (vertical) direction. The typical value of the maximum power density is $400 \mathrm{~kW} / \mathrm{mrad}^{2}$. At a typical distance of $20 \mathrm{~m}$ from the light source (ID) to the XBPM, the beam sizes are 2 and $0.8 \mathrm{~mm}$ HWHM in the horizontal and vertical directions, respectively, and the maximum power density is $1 \mathrm{~kW} / \mathrm{mm}^{2}$.

Therefore, the four detection elements are located at $\pm 3.5 \mathrm{~mm}$ horizontally and $\pm 2.0 \mathrm{~mm}$ vertically from the center of the beam axis such that they are not exposed to an SR beam with a power density of $10 \mathrm{~kW} / \mathrm{mrad}^{2}$ $\left(25 \mathrm{~W} / \mathrm{mm}^{2}\right.$ at the XBPM position) or more. It is estimated that the power that is irradiated constantly during operation is $10 \mathrm{~W}$ or less in the ID-BL, and that it could be temporarily increased to approximately $25 \mathrm{~W}$ during the commissioning or tuning.

To evaluate the heat transfer characteristics of this monitor, the thermal finite element analysis (ANSYS ${ }^{\circledR}$ Release 17.0) was carried out on the diamond heat sink $\left(20 \times 8 \times 0.3 \mathrm{~mm}^{3}\right)$, the heat sink holder, and the cooling base. In the quarter model used for the analysis, the heat sink holder and the cooling base are treated as a monolithic cylinder for simplification. Figure 9 shows an analysis

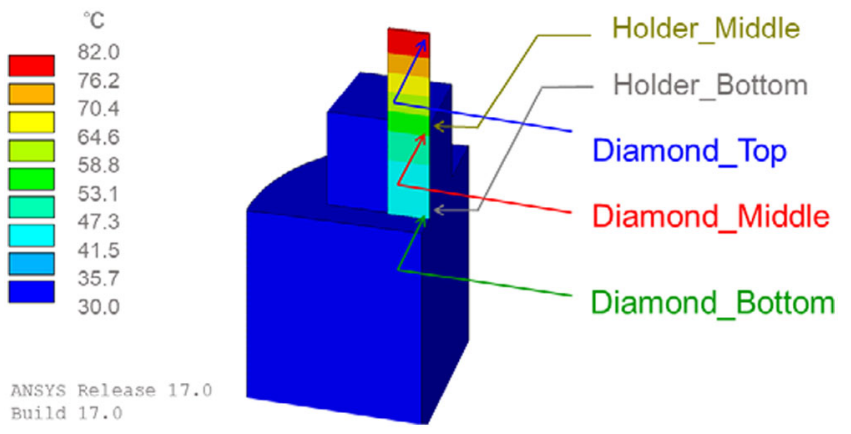

FIG. 9. Quarter model used for the thermal finite element analysis, where the heat sink holder and the cooling base are treated as a monolithic cylinder. The analysis result of a typical case in the normal operation of the SPring-8 standard ID-BLs is shown in a contour diagram. 


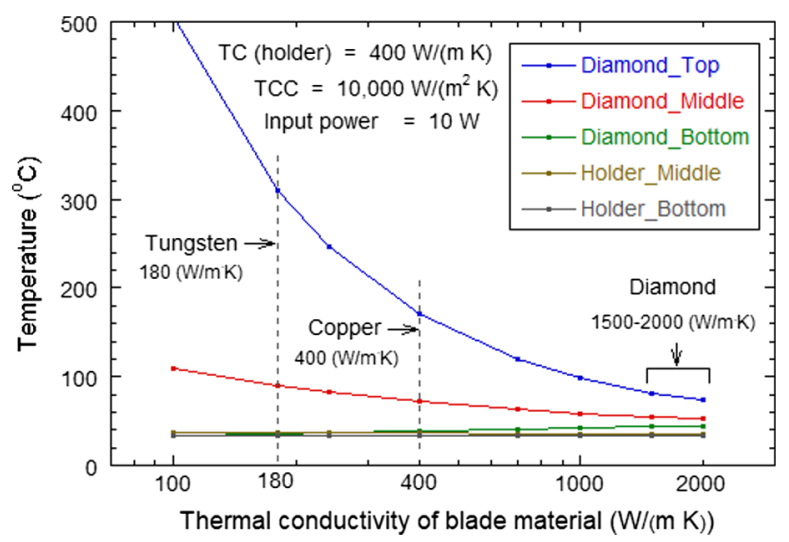

FIG. 10. Temperature variations of the quarter model due to difference in the TCs of blade materials.

result of a typical case in the normal operation of the SPring-8 standard ID-BLs. The thermal conductivity (TC) of diamond and copper are set to 1,500 and $400 \mathrm{~W} /(\mathrm{m} \mathrm{K})$, respectively. Thermal contact conductance (TCC) between the diamond heat sink and the copper heat sink holder is assumed to be $10,000 \mathrm{~W} /\left(\mathrm{m}^{2} \mathrm{~K}\right)$ in this calculation. The input power of $10 \mathrm{~W}$, which is equivalent to the maximum value assumed in the normal operation, is distributed to the tip $\left(1 \times 8 \times 0.3 \mathrm{~mm}^{3}\right)$ of the heat sink with uniform heat flux. The homothermal condition $\left(30^{\circ} \mathrm{C}\right)$ is set at the bottom of the cooling base. In this typical case, the temperature of the top of the diamond heat sink will be $82^{\circ} \mathrm{C}$, which is well tolerated for use.

Figure 10 shows temperature variations in the quarter model due to difference in the TCs of blade materials used for the heat sink under the condition that the TCC is $10,000 \mathrm{~W} /\left(\mathrm{m}^{2} \mathrm{~K}\right)$. If the TC of diamond is higher than $1,500 \mathrm{~W} /(\mathrm{m} \mathrm{K})$, the temperature rise is moderately suppressed suggesting that the exact value of the TC of diamond is not strictly needed. In contrast, if copper or tungsten plates are used for the heat sink, the temperature rise exceeds acceptable limits.

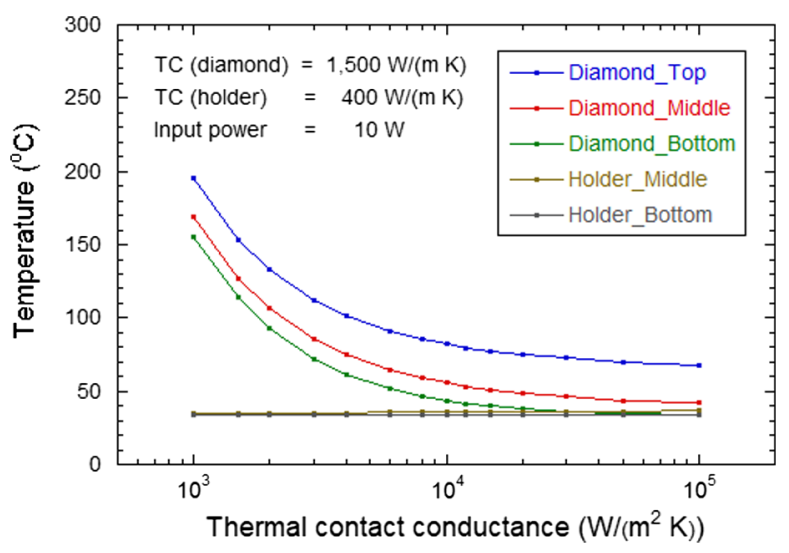

FIG. 11. Temperature variations of the quarter model due to difference in the TCC between diamond and copper.

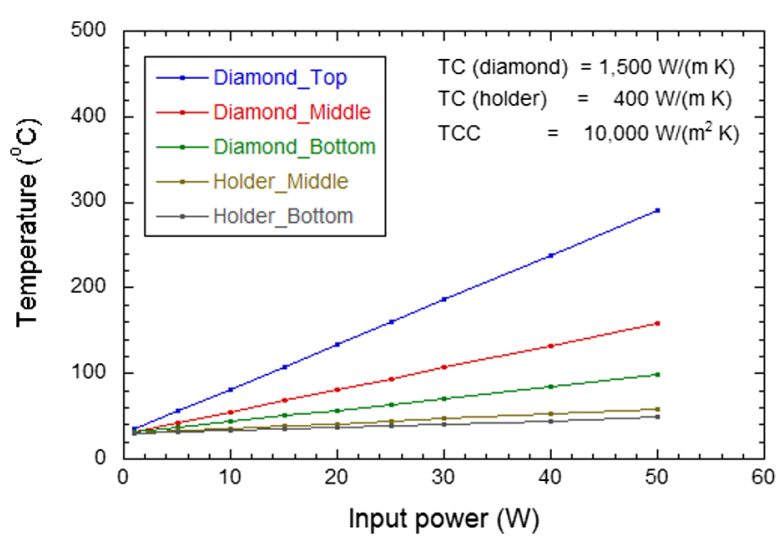

FIG. 12. Temperature variations of the quarter model due to difference in input power. TCC between diamond and copper is assumed to be $10,000 \mathrm{~W} /\left(\mathrm{m}^{2} \mathrm{~K}\right)$.

As it is not easy to estimate precisely the TCC between the diamond heat sink and the copper heat sink holder, the temperature variations in the quarter model were investigated by changing the TCC. Figure 11 shows that the temperature variations are considerably low if the TCC exceeds $10,000 \mathrm{~W} /\left(\mathrm{m}^{2} \mathrm{~K}\right)$, and that the temperature rise exceeds the tolerance of $100{ }^{\circ} \mathrm{C}$ if the TCC is below $2,000 \mathrm{~W} /\left(\mathrm{m}^{2} \mathrm{~K}\right)$.

The maximum input power is expected to reach approximately $25 \mathrm{~W}$ during commissioning/tuning of the beam lines. The temperature variations of the quarter model were investigated by changing the input power, as shown in Fig. 12.

The estimated maximum temperatures that are attained owing to the different heat sink materials, which are described earlier, are summarized in Table I. Thus, the proposed design with diamonds for the heat sink can be used.

\section{Considerations on the TCC}

Cooling the diamond heat sink is an important technical factor for enhancing the heat transfer characteristics. First, we examined the brazing joints that have the highest TCC. Based on the result of a bonding test using a sample of diamond plate and a tungsten block, we inferred that

TABLE I. Maximum temperature attained owing to different heat sink materials (estimated). Values denoted with $\dagger$ are not indicated in Figs. 10-12.

\begin{tabular}{lccc}
\hline \hline $\begin{array}{l}\text { Material of } \\
\text { heat sink }\end{array}$ & $\begin{array}{c}\text { Thermal conductivity } \\
{[\mathrm{W} /(\mathrm{m} \mathrm{K})]}\end{array}$ & $\begin{array}{c}\text { Input } \\
\text { power }[\mathrm{W}]\end{array}$ & $\begin{array}{c}\text { Maximum } \\
\text { temperature }\left[{ }^{\circ} \mathrm{C}\right]\end{array}$ \\
\hline Tungsten & 180 & 10 & 310 \\
& & 25 & $730^{\dagger}$ \\
Copper & 400 & 10 & 171 \\
& & 25 & $382^{\dagger}$ \\
Diamond & \multirow{2}{*}{$1500(2000)$} & 10 & $82\left(74^{\dagger}\right)$ \\
& & 25 & $160\left(139^{\dagger}\right)$ \\
\hline \hline
\end{tabular}


brazing directly to diamond is difficult because the diamond is easily cracked and damaged during brazing, and the poor vacuum of a brazing chamber causes impurity deposition and a deterioration in the electrical insulation of the diamond. Next, a method of inserting an indium foil (In, melting point $156^{\circ} \mathrm{C}$ ) or a tin foil $\left(\mathrm{Sn}\right.$, melting point $\left.228^{\circ} \mathrm{C}\right)$ between a diamond plate and a copper block was examined. Each foil was separately sandwiched between the diamond plate and the copper bock, heated to a temperature of approximately $10^{\circ} \mathrm{C}$ above the respecting melting point of the foil material, and finally, cooled to room temperature $\left(25^{\circ} \mathrm{C}\right)$ for observation. We observed that the adhesion between the copper and the indium foil and between the diamond and the tin foil were satisfactory. However, the observation did not indicate whether using either foil was appropriate. Therefore, we decided to maintain the vacuum bake-out temperature under $180^{\circ} \mathrm{C}$ to prevent damage and then selected indium, whose melting point is close to the bakeout temperature, as the thermal interface material.

\section{PERFORMANCES IN PULSE MODE}

\section{A. Structure}

In the conventional XBPMs, as the blade-shaped detection elements are mounted parallel to the beam axis, most of the photoelectrons are generated at the end face of the blades irradiated with the SR beam. The detection elements of a prototype pulse-mode XBPM are designed to have an inclination of approximately $1 / 20$ with respect to the beam axis so that it is irradiated mainly on one side of the blade. Consequently, an electric field applied between the charge collecting electrode and the detection elements becomes uniform, and the photoelectron emission is efficiently controlled. Figure 13 shows the heat sink holder and the detection elements used in the actual devise. Titanium, which acts as the photocathode, is deposited on one side of all detection elements. Figure 14 shows a signal transmission line with the microstripline structure. Figure 15 shows the overall structure of this monitor. The heat sink holder (Fig. 13) and the signal transmission line (Fig. 14) are combined in the six-way cross chamber. A shading mask is fixed immediately upstream of the six-way cross

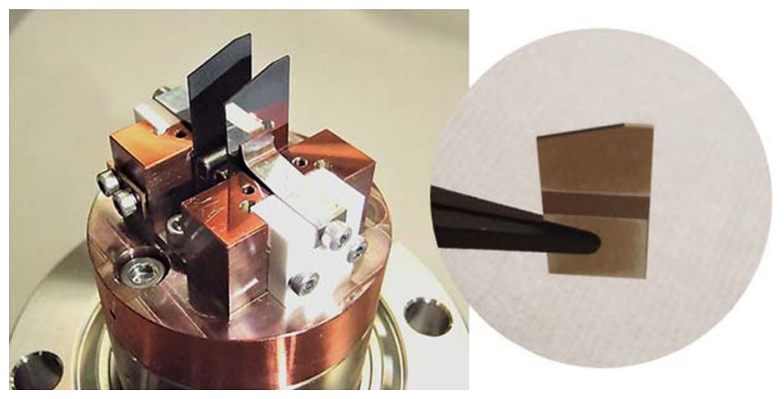

FIG. 13. Photographs of the heat sink holder (left) and the detection elements (right).

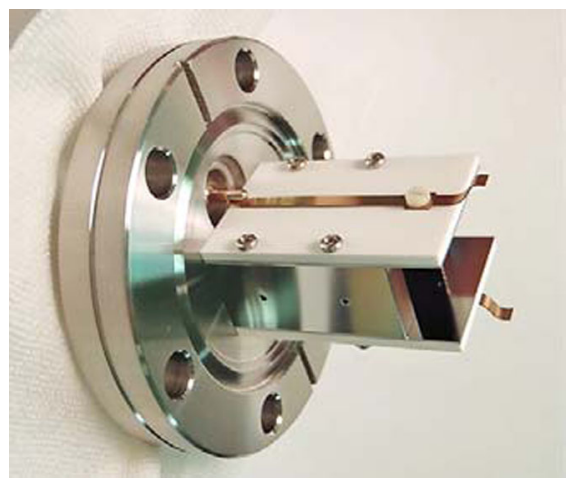

FIG. 14. Photograph of a signal transmission line with the microstripline structure.

chamber to prevent the heat sink holder from irradiation, which is widely emitted from the bending magnet on the upstream and downstream of the insertion device.

By fixing a couple of charge collecting electrodes (retarding electrodes) on the left and right sides of the
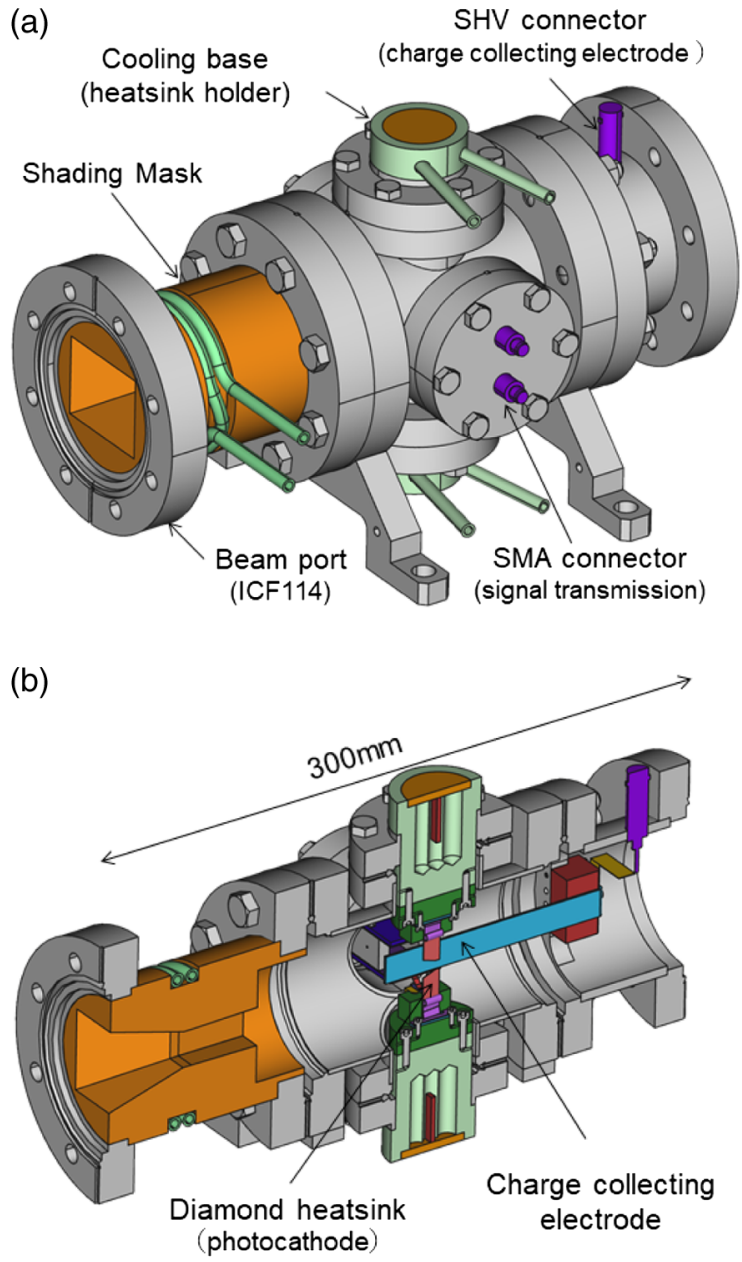

FIG. 15. Overall structure of this monitor, (a) a view of the external configuration and (b) a view of the internal configuration along a vertical cross section. 


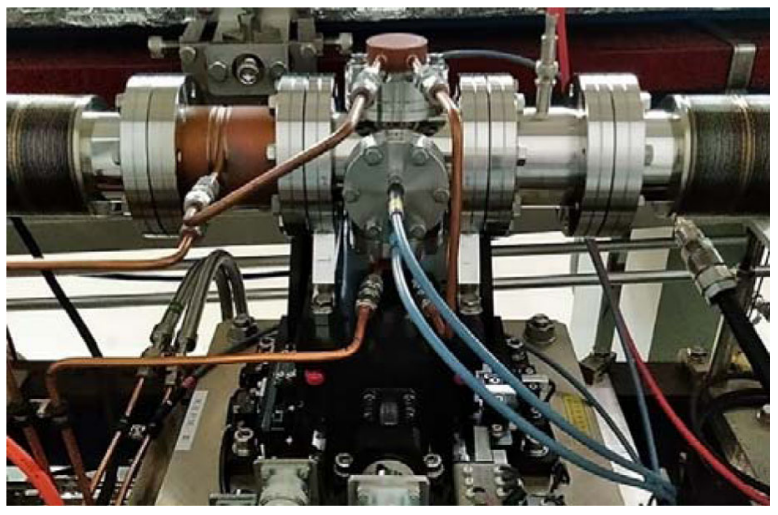

FIG. 16. Photograph of a pulse-mode XBPM installed in the SPring-8 BM-BL (BL02B1) front end. The monitor is mounted on a XY stage to be moved horizontally and vertically. The beam direction is from left to right.

detection elements, the photoelectrons emitted from the detection elements into the vacuum are enhanced (suppressed) when negative (positive) voltage is applied. In the direct current (dc) mode measurement, same as the conventional XBPMs, the output signal becomes stable, and accuracy of the monitor is improved by applying a voltage of approximately $+100 \mathrm{~V}$ to the charge collecting electrodes. As will be described later, in the pulse-mode measurement, the emission of low energy photoelectrons, which disrupt the pulse waveform, is suppressed by applying a negative voltage; hence, optimizing the pulse waveform. This monitor was installed in the SPring-8 BM-BL (BL02B1) front end (Fig. 16), and performance tests were carried out. Among four signal cables, Sucofeed 1/2" (HUBER + SUHNER), which has small attenuation in high-frequency region, was used as the UL cables to observe pulse waveform. For the remaining three cables (UR, LL and LR), S-04272B (HUBER + SUHNER) was used, because they are primarily used in the dc mode measurement.

\section{B. Observation of pulse waveforms}

Figure 17 shows a typical pulse waveform of the current signal on this monitor. The filling pattern of the storage ring was " $1 / 7$ filling +5 bunches" mode. One of the five bunches was observed. A pulse length with a FWHM of $0.7 \mathrm{~ns}$ was obtained when the applied voltage of the charge collecting electrodes was $-100 \mathrm{~V}$. Compared to the pulse waveform R-5, shown in Fig. 8, the pulse length is slightly wider, and the tip has a double peak structure. It was considered that a long tiny ringing remained after the pulse was derived from the high-frequency resonance generated in the vacuum chamber.

As shown in Fig. 18, several pulse waveforms are observed by changing the applied voltage. The pulse waveform features, such as the height and length, significantly depend on polarity and amplitude of the applied voltage. The pulse height and width decrease as a negative

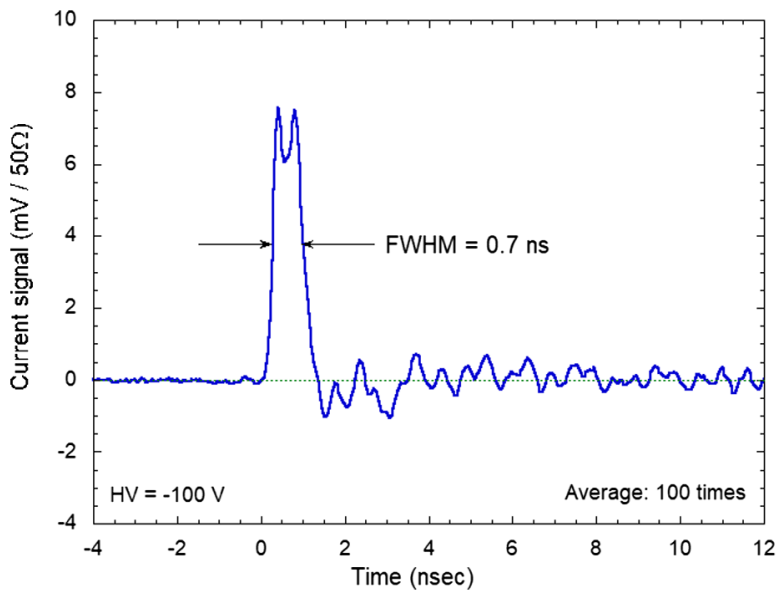

FIG. 17. Typical pulse waveform of the current signal. An oscilloscope (4 GHz B. W., $20 \mathrm{GS} / \mathrm{s}, 100$-time average) is used for pulse waveform observation. The baseline is marked with a dotted line.

voltage is applied because it blocks the emission of negatively charged photoelectrons. However, as the negative voltage is increased, the double peak structure of the pulse waveform becomes more prominent, which cannot be explained by the resonance of the high-frequency component in the vacuum vessel because a resonance should decay gradually over time. The first peak in this doublepeak structure is due to the photoelectrons that have sufficiently high energy to not be affected by the voltage of the charge collecting electrode. The second peak is due to the deceleration of the low energy photoelectrons by the negative voltage of charge collecting electrode.

By setting the applied voltage to $-40 \mathrm{~V}$ or less, the tail decreases and the FWHM becomes 1 ns or less. This applied voltage range is suitable for a shot-by-shot measurement with narrow pulse intervals. Conversely, by setting the applied voltage to $-40 \mathrm{~V}$ or higher, the tail becomes bigger and the pulse length increases. As the applied voltage exceeds $+100 \mathrm{~V}$, the signal charge becomes

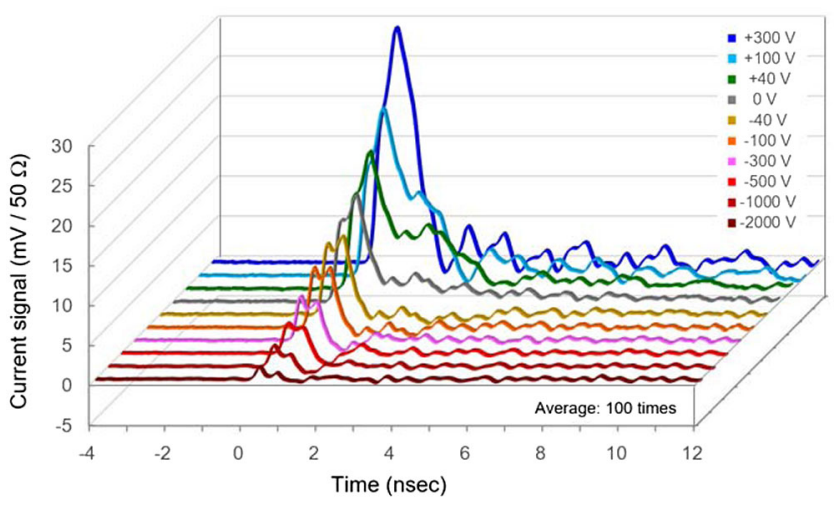

FIG. 18. Variation of pulse waveforms observed by changing the voltage of the charge collecting electrodes. 
constant and the fall time reduces; however, the ringing with a large amplitude in the tail becomes noticeable. The pulse height further increases and the ringing intensifies above $+300 \mathrm{~V}$. Thus, an applied voltage range of from +100 to $+300 \mathrm{~V}$ is suitable for an isolated single-shot measurement. The relationship between the applied voltage and the signal current in the dc mode is described in Sec. IVA.

\section{Pulse height response to $x$-ray incidence}

To evaluate the response of the output signal of the detection element, it is appropriate to inject the storage ring with a filling pattern in which the pulse intensity of each bunch is modulated. This method was adopted to evaluate a microstripline detection element [19]. However, as this filling pattern was not available in the SPring-8 user operation mode, this monitor was evaluated in the $1 / 7$ filling +5 bunches condition, which is one of the user operation modes, as a simple alternative method.

The response of the pulse height to incident $\mathrm{x}$ rays was determined as follows. As shown in Fig. 19, the pulse height of the pulse train part of the filling pattern $1 / 7$ filling +5 bunches is compared with the bunch current of the storage ring, which is proportional to the x-ray intensity. The bunch current was measured with the sum signal from a button BPM pickup, calibrated with the storage ring current monitor (dc current transformer). In the vertical
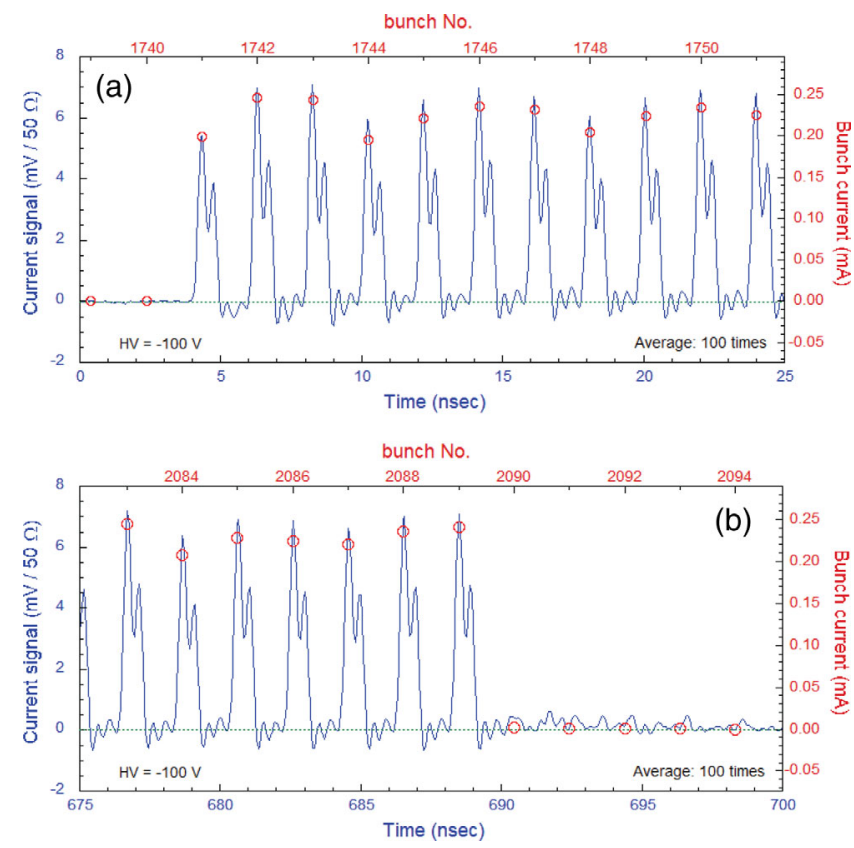

FIG. 19. Response of the pulse height to incident $\mathrm{x}$ rays, (a) head of pulse train, (b) tail of pulse train. The pulse waveform is indicated by the blue line and the bunch current is indicated by the red circles. The baseline is marked with a dotted line. The applied voltage of the charge collecting electrodes is set to $-100 \mathrm{~V}$. axis, the bunch current (right) is scaled to match the pulse height (left) of the leadoff pulse. The pulse height tracks the bunch current of the storage ring [Fig. 19(a)]. However, the second and subsequent pulses are approximately $10 \%$ higher than the bunch current because the pulses are piled up on the tail of the preceding pulses, even in the middle part of the train, which is evident from the trailing after the last pulse of the pulse train [Fig. 19(b)].

The aforementioned measurement was made at the applied voltage $(-100 \mathrm{~V})$ of the charge collecting electrodes. Although the applied voltage was changed in various ways, no improvement in the pileup situation was observed. To reduce the pileup, it is necessary to suppress the tiny, yet long-lasting ringing, which is also shown in Fig. 17. The ringing is considered to be generated because of the high-frequency resonance in the vacuum chamber, therefore it must be removed by the available methods, such as the insertion of an absorber (like silicon carbide).

\section{Synthesization of position sensitive signal}

Based on the unipolar single pulse output of this monitor, we devised a new method to synthesize position sensitive signal. In case of beam position monitor for charged particles, position calculation is performed after digital conversion of the pulse signals from respective detection electrodes. However, for this monitor, the position sensitive signal is synthesized from unipolar single pulses only by using a simple rf circuit.

Figure 20(a) shows the configuration of the rf circuit to synthesize the position sensitive signal. The UR signal is split into two signals by a resistive power divider: one is connected to the oscilloscope, and the other is reversed in phase at the short end of the tip of the stub cable. In this measurement, the applied voltage is set to $-300 \mathrm{~V}$, because the tail of the original waveform, which is obtained before connecting the rf circuit, is sufficiently suppressed at below this voltage, as shown in Fig. 20(b). It is not identical to that measured at $-300 \mathrm{~V}$, as shown in Fig. 18, due to the difference in the cables used. The LR signal is kept at the same phase by setting the tip of the stub cable to the open end. A "difference" signal is synthesized by combining the inverted UR signal and the phase pure LR signal. Figure 21(a) shows the experimental results. The pulse at $t=0 \mathrm{~ns}$ is a "sum" signal, in which the UR signal and the LR signal are combined via the shortest path. The pulse after approximately $8.6 \mathrm{~ns}$ is the difference signal, in which the UR signal and the LR signal are combined after being reflected at the tip of each stub cable. The pulse charge of the sum signal remains constant even when the monitor is scanned with $0.1 \mathrm{~mm}$ step in the vertical direction. However, the pulse height of the difference signal is sensitive to a change in the beam position. In Figure 21(b), the integrated charge of the difference signal at each position is plotted, which shows that the integrated charge is linearly proportional to the vertical stage position over an operating range of about $1 \mathrm{~mm}$. 

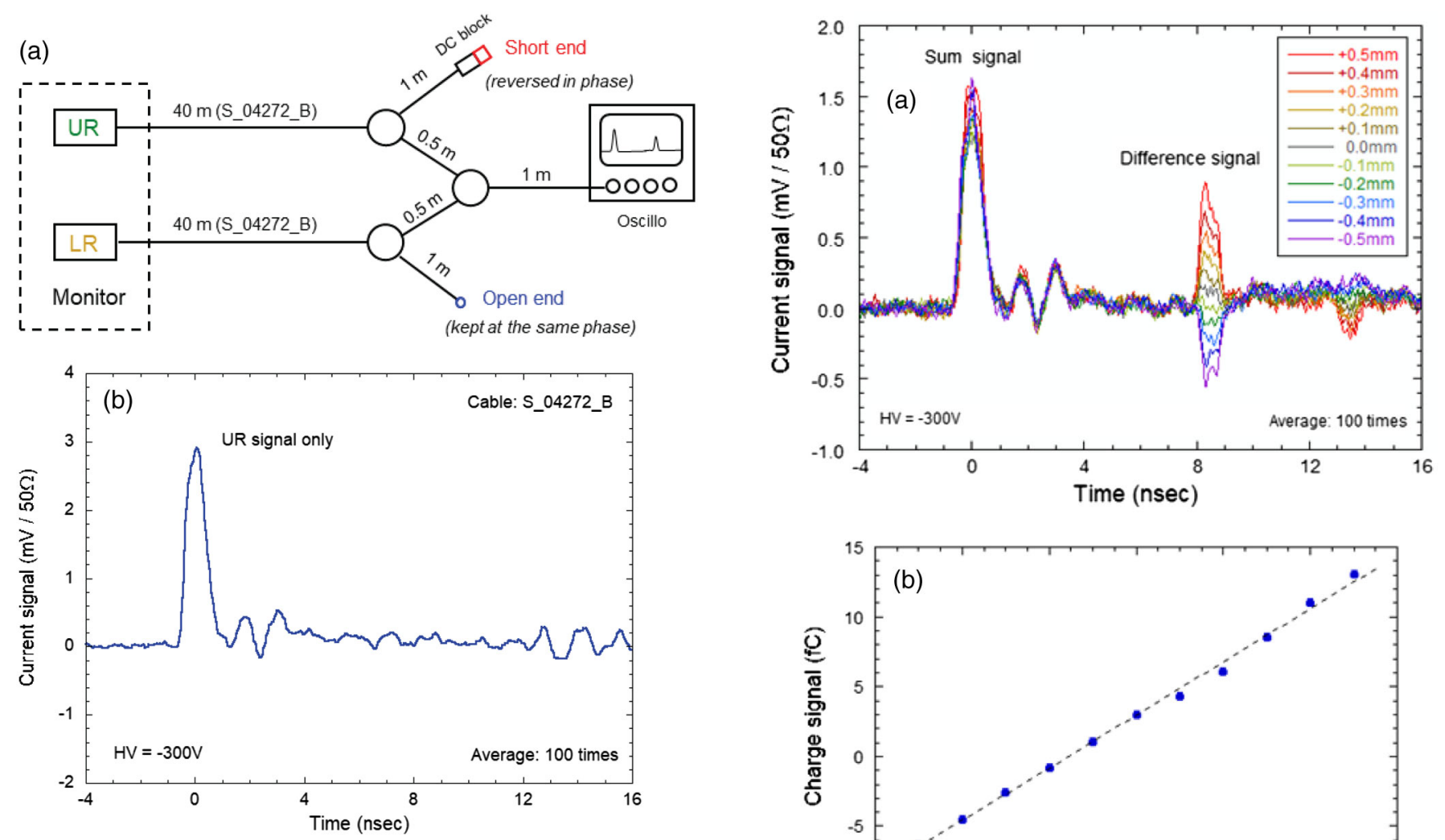

FIG. 20. (a) Configuration of the rf circuit to generate the position sensitive signal. Dividers $(-6 \mathrm{~dB})$ are shown as open circles. (b) Typical pulse waveform of the current signal. The used cable is S_04272_B (HUBER + SUHNER).

\section{E. Single pulse resolution}

To comprehend the characteristics of the waveforms, the pulse-mode signal waveforms that are introduced in the evaluation tests are averaged 100 times over the entire data range. The averaging is performed because the evaluation tests were conducted in the BM-BL, which has a much smaller current signal than the ID-BLs, and the pulse height fluctuates significantly in the single-shot measurement. Presently, this prototype fails to exhibit the required resolution in the tests performed in the BM-BL. For example, at the applied voltage of $+100 \mathrm{~V}$, each integrated value of the UR or LR pulse signal (typically $\sim 0.8 \mathrm{pC}$ ) is observed with a typical fluctuation of from 1.7 to $2.1 \% \mathrm{rms}$. The error propagation in the operation of "difference/sum" of four signals is estimated to be approximately $1.4 \% \mathrm{rms}$, which corresponds to approximately $20 \mu \mathrm{m} \mathrm{rms}$ when converted into an error in beam position estimation. The conversion formula and coefficient $(A y=1.25)$ are described later. The current signals observed in the ID-BLs are increased to be 30 times that in the BM-BLs; therefore, in general, we can estimate the resolution as $1 /$ several (1/the square root of 30$)$. Owing to the increase in the current signals, a resolution of approximately $4 \mu \mathrm{m}$ rms is expected when the signal is measured individually for each detection element and the beam

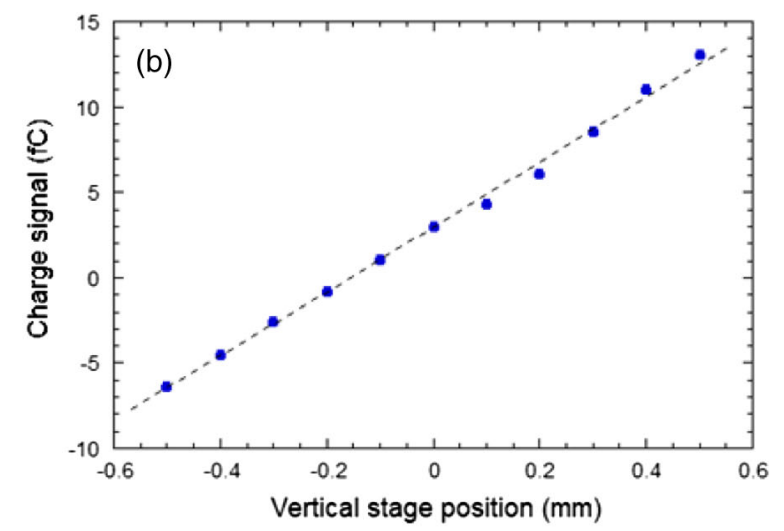

FIG. 21. (a) Variation of position sensitive signals. (b) Plot of the integrated charge of the difference signal against the vertical stage position is plotted.

position is converted. On the contrary, when the rf circuit was used to synthesize the position sensitive signal, described in Sec. III D, the resolution deteriorated further because the pulse height was reduced to $1 / 8$ of its initial value by passing it through the resistive power dividers $(-6 \mathrm{~dB}) 3$ times. Through this study, we have confirmed that the fundamental solution to improve the resolution requires an increase in signal charge. To achieve this, it is necessary to increase the size of the detection element as much as possible within a range such that its highfrequency performance is not affected, or we can use diamond as a semiconductor detector.

\section{EVALUATION IN de MODE}

Operating in the dc mode, the existing conventional XBPMs in the SPring- 8 constantly monitor the short- and long-term photon beam positions, and the beam vibration, typically, at frequencies lower than $500 \mathrm{~Hz}$. Although this pulse-mode monitor is optimized for pulse-mode measurements, it can be also used in the dc mode similar to conventional XBPMs. A thorough evaluation in the dc mode confirms that this monitor operates properly in the 
pulse mode. Additionally, to demonstrate the pulse-mode $\mathrm{XBPM}$ in the SPring-8 BLs during a user operation, it is necessary to replace the conventional XBPMs, which are already in operation, with the newly designed XBPM owing to the limitation in space availability. Therefore, it is crucial that the newly designed XBPM, as an alternative to the conventional XBPM, operates normally.

The characterization tests of the pulse-mode monitor in the dc mode are described in this section. The current signals from the blades are measured using a four-channel current-to-voltage converter in the frequency range used in a conventional XBPM (from dc to $10 \mathrm{kHz}$ ). The data acquisition rate of the analog to digital converter (ADC) was one data point per $6 \mathrm{~s}$ with a time constant of approximately $1 \mathrm{~s}$.

\section{A. Variation of current signal with applied voltage}

Observing the current signal that depends on the applied voltage of the charge collecting electrode is useful to conduct a simple operational check of the XBPM. Figure 22 shows the variation of the average of four current signals in the dc mode and the signal charge in pulse mode with respect to the voltage applied to the charge collecting electrodes. Evidently, they are highly dependent on the voltage of the charge collecting electrodes. The slope is steep around $0 \mathrm{~V}$, and the retarding effect is obvious on the negative side because the blades of this monitor are not exactly parallel to the beam axis and are slightly inclined, such that only one side of the blade is illuminated, which enhances the effective electric field. As a result, it is possible to suppress both the emission of slow electrons having an energy of several tens of electron volts, and the increase in pulse length. For applied voltages above $+40 \mathrm{~V}$, the current signal is constant. This is consistent

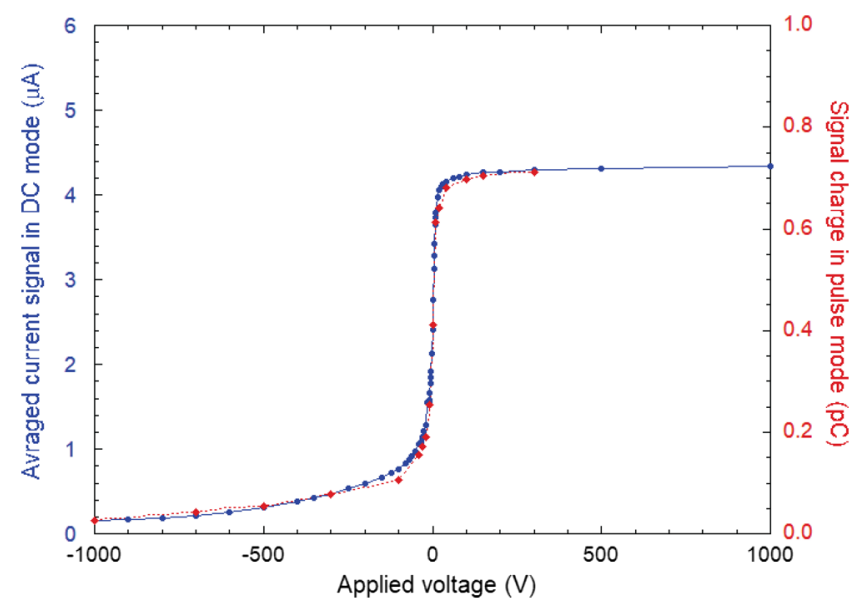

FIG. 22. Variation of the average of four current signals in dc mode and the signal charge in the pulse mode with respect to the voltage applied to the charge collecting electrodes. The signal charge in the pulse mode (red dots) is the integral of the pulse waveform in Fig. 18. with the phenomenon, as shown in Fig. 18, that the integrated charge becomes constant (the pulse width decreases while the pulse height increases) for an applied voltage of $+40 \mathrm{~V}$ and above. Near $0 \mathrm{~V}$, the signal charge in the pulse mode is slightly lower than that in the dc mode. This is because the signal charge is suppressed by the space-charge effect of the photoelectrons, which are emitted by a single pulse with a high charge. In the dc mode operation, the applied voltage should be $+40 \mathrm{~V}$ or higher, which is the plateau region shown in Fig. 22. Subsequent evaluation tests in the dc mode are conducted at the applied voltage of $+100 \mathrm{~V}$.

\section{B. Position sensitivity and resolution}

Vertical scan measurements were performed to determine the sensitivity, resolution and linearity region of this monitor. Figure 23 shows the results of the scan measurement with scan steps of $0.2 \mathrm{~mm}$. A vertical correction coefficient, Ay, of the value 1.25 was obtained from the slope of the difference/sum of $y$, which is linear over a wide range of the vertical stage position from -0.5 to $+0.5 \mathrm{~mm}$. This corresponds to the linear region in the pulse mode, as shown in Fig. 21(b). In contrast, the difference/sum of $\mathrm{x}$ is maintained at a constant value because the SR beam from the bending magnet spreads uniformly in the horizontal direction. By evaluating the horizontal beam position, it can be confirmed that the four detection elements are operating normally.

Figure 24 shows the results of the scan measurement in steps of $1 \mu \mathrm{m}$ at $1 \mathrm{~min}$ intervals to evaluate the resolution. A resolution of $0.12 \mu \mathrm{m}$ rms was obtained from the measurement results. It was shown that in the dc mode, this monitor exhibited a resolution that is comparable to that of the conventional XBPM.

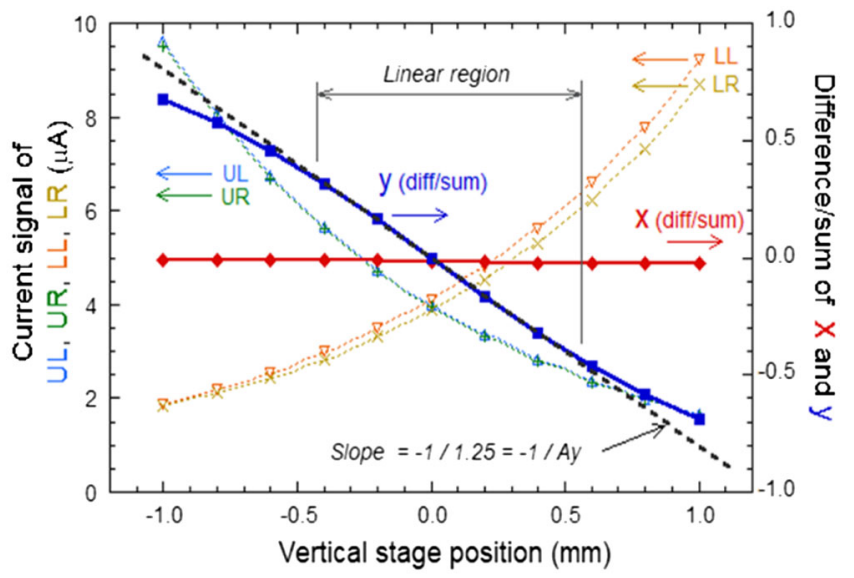

Difference/sum of $x=\frac{(U R+L R)-(U L+L L)}{U L+U R+L L+L R}$, Difference/sum of $y=\frac{(U L+U R)-(L L+L R)}{U L+U R+L L+L R}$

FIG. 23. Results of the scan measurement with scan steps of $0.2 \mathrm{~mm}$. 


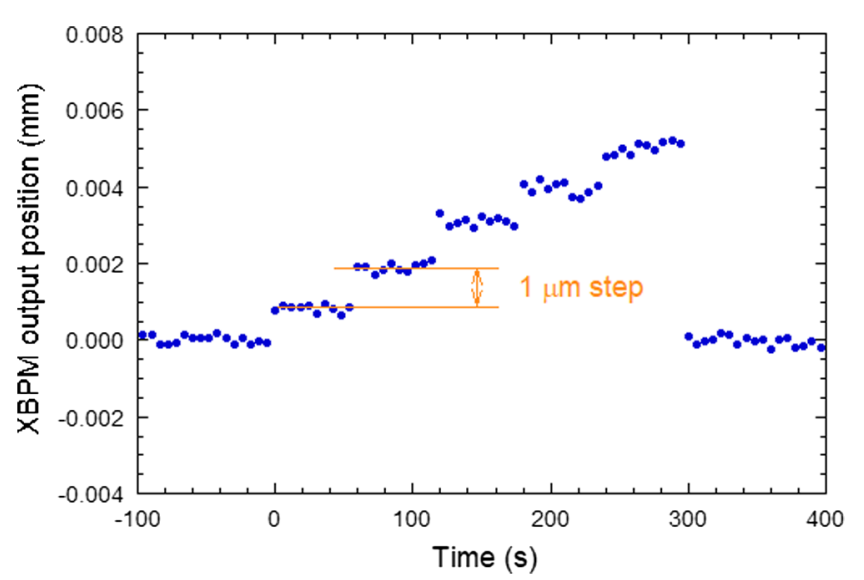

FIG. 24. Results of scan measurement with scan steps of $1 \mu \mathrm{m}$.

\section{Proportionality of current signal}

The proper operation of this monitor can be checked by evaluating the response of each current signal as the temperature of the detection element changes drastically. Figure 25(a) shows the variation of the sum of the four
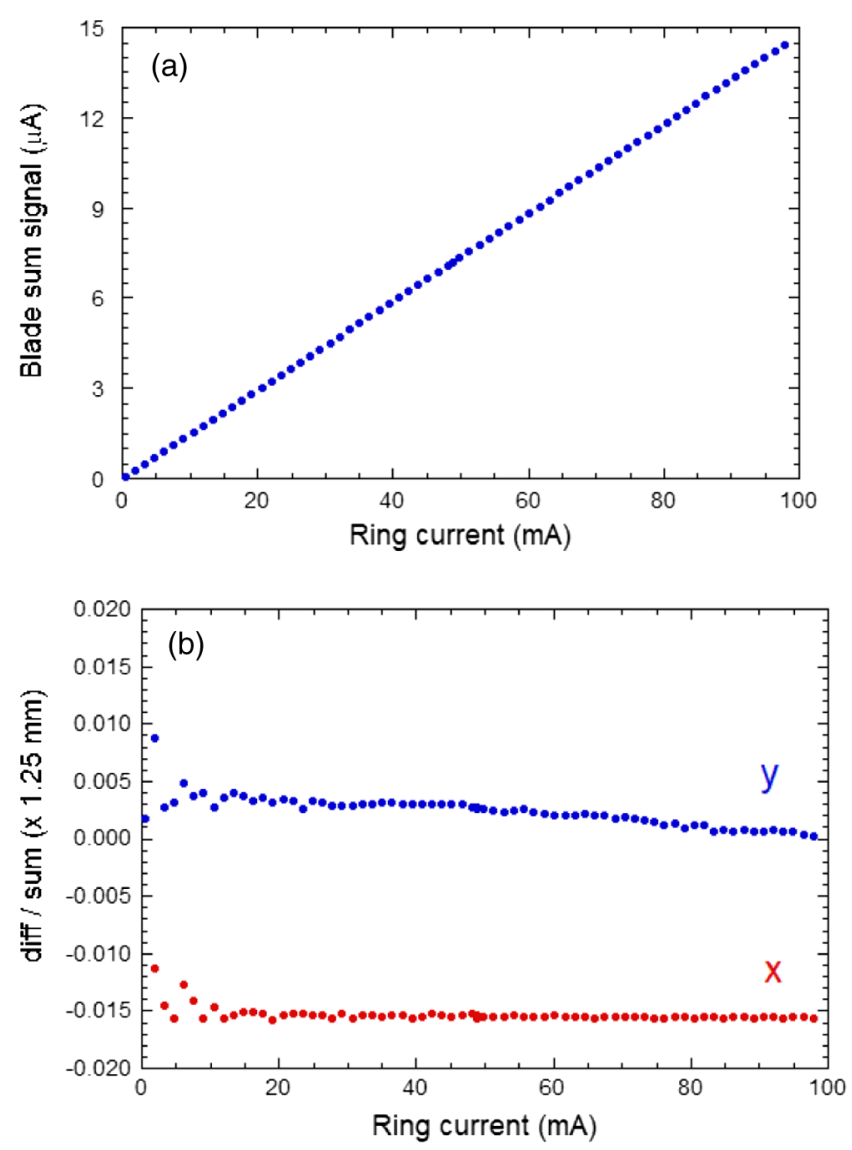

FIG. 25. (a) Variation of the sum of four blade signals with respect to the storage ring current. (b) Plot of the calculated values of difference/sum of the four blade signals with respect to the storage ring current in the horizontal (x) and vertical (y) directions. blade signals with the storage ring current during beam injection from 0 to $100 \mathrm{~mA}$. Evidently, the dc signal from the blades is approximately linearly proportional to the ring current.

As the orbit of the electron beam is not corrected by a feedback while the storage ring is injected, it is expected that the electron beam orbit, which determines the SR beam axis, will fluctuate to some extent during the injection. Therefore, we investigated the horizontal and vertical beam positions derived by this XBPM operating in the dc mode. Figure 25(b) shows the variation of the calculated values of difference/sum of the four blade signals with the ring current in the horizontal and vertical directions. As the ring current increases, a significant displacement of approximately $4 \mu \mathrm{m}$ in the difference/sum blade signal value in the vertical directions was observed. In this experiment as well, the horizontal difference/sum remained constant, which confirmed that it was operating normally.

These experimental results show that the dc mode operation works properly, even with varying thermal loads. This is a prerequisite for a successful operation in the pulse mode.

\section{Long-term stability}

A reliable evaluation of the stability of the XBPMs can be achieved by measuring the dynamics of a perfectly stable SR beam; however, this is practically impossible. Therefore, the stability is evaluated as follows: The pulsemode XBPM is equipped with four detection elements (UL, UR, LL, and LR). Here, the UL and LL pair (left XBPM) and the UR and LR pair (right XBPM) are treated as two independent XBPMs that monitor the beam in the vertical direction (Fig. 26). As the SR beam from the bending magnet spreads uniformly in the horizontal direction, these two independent XBPMs exhibit the same result. Therefore, the stability can be evaluated by simultaneously

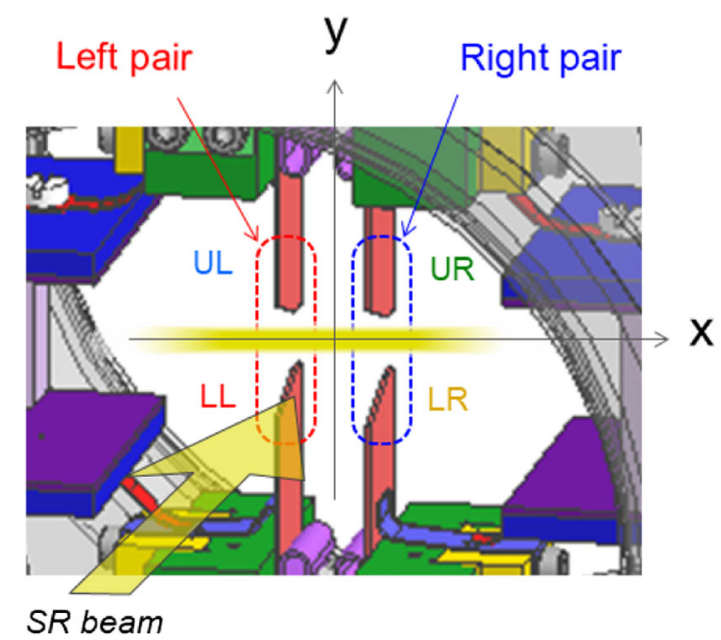

FIG. 26. Schematic diagram of a pair of left and right XBPMs for vertical measurements. 


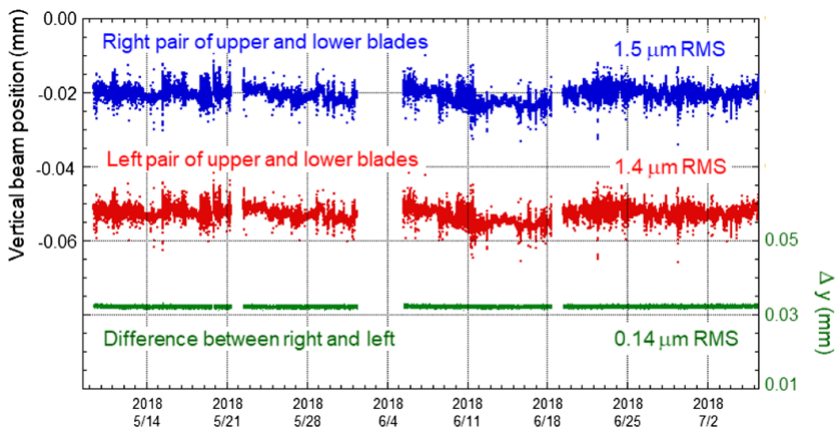

FIG. 27. Trend graph over two months of the vertical position from the left and right XBPMs and the difference between them.

observing and comparing their outputs. In principle, the calculated vertical positions are expected to exhibit identical behavior in both the left and right XBPMs. We tried to evaluate the long-term stability of this monitor by comparing the output values of both of the XBPMs. Figure 27 shows a trend graph over two months of the vertical position from the left and right XBPMs and the difference between them. The standard deviations of the left and right XBPM output values were 1.4 and $1.5 \mu \mathrm{m}$, respectively. These values are mainly due to actual beam displacements. In addition, a difference of $0.14 \mu \mathrm{m}$ rms between the output values of the XBPMs, which corresponds to the resolution of the XBPMs, as mentioned in Sec. IV B, was observed over the period. Evidently, a considerable stability over a period of two months has been demonstrated.

As described earlier, the stability was confirmed by acquiring data continuously for two months, sampling at $6 \mathrm{~s}$ intervals in the dc mode operation; thus, it can be concluded that the newly designed XBPMs showed stable operation, both for short and long terms. From this, it can be inferred that the operation in the pulse mode is also stable in the long term. In fact, this monitor operated stably in the pulse mode for three years.

\section{CONCLUSION}

We have designed and manufactured a pulse-mode XBPM prototype using diamond heat sinks aiming to use it in the ID-BLs in a synchrotron radiation facility.

The high-frequency measurements on the test unit showed that a subnanosecond unipolar single pulse was obtained by using the microstripline structure for the signal transmission lines. The thermal finite element analysis confirmed that the proposed design could be used if the TCC between the diamond heat sink and the cooling holder was assumed to be $10,000 \mathrm{~W} /\left(\mathrm{m}^{2} \mathrm{~K}\right)$.

Performance tests were carried out at the SPring-8 BMBL (BL02B1) front end. A unipolar single pulse of $0.7 \mathrm{~ns}$ FWHM was obtained. We qualitatively explained the appearance of the double peak in the pulse. The pulse waveform was controlled by varying the voltage applied to the charge collecting electrodes. The height of each pulse was reflected in the bunch current of the storage ring. We demonstrated that the directly readable position sensitive signal was generated by synthesizing reflected pulses from the open end and the short end of the stub cables. Currently, the single pulse resolution that can be obtained from the evaluation test in the BM-BL is estimated to be approximately $20 \mu \mathrm{m}$. We also explained that the required resolution can be improved if the test is conducted in the ID-BLs, where the SR beam intensity is extremely strong. The fundamental measure to improve the resolution is to increase the quantum efficiency of the detection element itself by increasing the detector size or by using a diamond semiconductor. It can be also expected that the resolution will be substantially improved if the long-lasting ringing is suppressed by inserting an absorber in the vacuum chamber. In addition, a data collection system with a high-speed ADC is indispensable for conducting systematic evaluation tests in pulse-mode operation.

The proper operation of this monitor was identified in the dc mode evaluation tests. We confirmed that the behavior of the signal charge with respect to the applied voltage of the collection electrodes in the pulse mode matched that in the dc mode. In the dc mode operation, a correction coefficient Ay of 1.25 was calculated from the vertical scan measurements, and a resolution of $0.12 \mu \mathrm{m} \mathrm{rms}$ was obtained. The dc signal from the blades was found to be linearly proportional to the bunch current. Finally, long-term stability of the proposed monitor was verified. These dc mode evaluation tests showed that this monitor meets the indispensable conditions for stable operation in the pulse mode. The monitor has been tested over a duration of three years, which establishes its durable and stable performance. Thus, the proposed monitor can be further developed for advance operations.

\section{ACKNOWLEDGMENTS}

The authors would like to thank T. Nakamura, K. Kobayashi, S. Kimura, and H. Osawa of the Japan Synchrotron Radiation Research Institute (JASRI) for their useful advice regarding high-frequency analysis. The design and fabrication of the pulse-mode XBPM has been done in cooperation with the Vacuum and Optical Instruments (Shinku-Kogaku, Inc., Tokyo, Japan). This work was supported by Japan Society for the Promotion of Science through a Grant-in-Aid for Scientific Research (c), No. 26390122 and No. 18K11943.

[1] D. Shu, B. Rodricks, J. Barraza, T. Sanchez, and T. M. Kuzay, The APS x-ray undulator photon beam position monitor and tests at CHESS and NSLS, Nucl. Instrum. Methods Phys. Res., Sect. A 319, 56 (1992).

[2] T. Mitsuhashi, A. Ueda, and T. Katsura, High-flux photon beam position monitor, Rev. Sci. Instrum. 63, 534 (1992). 
[3] W. B. Peatman and K. Holldack, Diagnostic front end for BESSY II, J. Synchrotron Radiat. 5, 639 (1997).

[4] H. Aoyagi, T. Kudo, and H. Kitamura, Blade-type x-ray beam position monitors for SPring-8 undulator beam lines, Nucl. Instrum. Methods Phys. Res., Sect. A 467, 252 (2001).

[5] E. van Garderen, J. Krempaský, M. Böge, J. Chrin, V. Schlott, T. Schmidt, and A. Streun, Characterization of the systematic effects of the insertion devices with photon beam position monitors, in Proceedings of the 2007 European Workshop on Beam Diagnostics and Instrumentation for Particle Accelerators, Venice, Italy, 2007 (JACoW, Venice, Italy, 2007), p. 126, TUPB26.

[6] C. Bloomer and G. Rehm, Operation of Diamond Light Source XBPMs with zero bias, in Proceedings of the 2nd International Beam Instrumentation Conference, Oxford, UK, 2013 (JACoW, Oxford, UK, 2013), p. 376, TUPC10.

[7] N. Yasuda, Y. Fukuyama, S. Kimura, K. Ito, Y. Tanaka, H. Osawa, T. Matsunaga, R. Kojima, K. Hisada, A. Tsuchino, M. Birukawa, N. Yamada, K. Sekiguchi, K. Fujiie, O. Kawakubo, and M. Takata, System of laser pump, and synchrotron radiation probe microdiffraction to investigate optical recording process, Rev. Sci. Instrum. 84, 063902 (2013).

[8] C. Moriyoshi, S. Hiramoto, H. Ohkubo, Y. Kuroiwa, H. Osawa, K. Sugimoto, S. Kimura, M. Takata, Y. Kitanaka, Y. Noguchi, and M. Miyayama, Synchrotron radiation study on time-resolved tetragonal lattice strain of BaTiO3 under electric field, Jpn. J. Appl. Phys. 50, 09NE05 (2011).

[9] Y. Fukuyama, N. Yasuda, H. Kamioka, J. Kim, T. Shibata, H. Osawa, T. Nakagawa, H. Murayama, K. Kato, Y. Tanaka, S. Kimura, T. Ohshima, H. Tanaka, M. Takata, and Y. Moritomo, Simultaneous measurements of picosecond lattice, and charge dynamics in Co-Fe cyanides, Appl. Phys. Express 3, 016601 (2010).

[10] N. Kumagai, H. Ohkuma, K. Soutome, M. Takao, and H. Tanaka, Orbit stabilization in SPring-8 storage ring, in Proceedings of the 18th Particle Accelerator Conference, New York, 1999 (IEEE, New York, 1999), p. 2340.

[11] S. Matsui, M. Oishi, H. Tanaka, T. Yorita, K. Tsumaki, N. Kumagai, and T. Nakazato, Orbit fluctuation of electron beam due to vibration of vacuum chamber in quadrupole magnets, Jpn. J. Appl. Phys. 42, L338 (2003).

[12] H. Ohkuma, S. Date, M. Masaki, T. Nakamura, T. Ohshima, K. Soutome, S. Takano, K. Tamura, M. Takao, H. Tanaka, and N. Kumagai, Beam-performance improvement of the SPring- 8 storage ring, in Proceedings of the 2003 Particle Accelerator Conference, Portland, OR (IEEE, New York, 2003), p. 881.

[13] H. Tanaka et al., Stable top-up operation at SPring-8, J. Synchrotron Radiat. 13, 378 (2006).
[14] T. Nakamura, S. Daté, K. Kobayashi, and T. Ohshima, Transverse bunch-by-bunch feedback system for the SPring-8 storage ring, in Proceedings of the 9th European Particle Accelerator Conference, Lucerne, 2004 (EPS-AG, Lucerne, 2004), p. 2646.

[15] T. Nakamura and K. Kobayashi, FPGA based bunch-by bunch feedback signal processor, in Proceedings of the 10th International Conference on Accelerator and Large Experimental Physics Control Systems, Geneva, Switzerland, 2005 (JACoW, Geneva, Switzerland, 2005), PO2.022-2.

[16] K. Kobayashi and T. Nakamura, New automatic bunch current sensitive fast attenuator for the rf front-end of bunch-by-bunch feedback system at SPring-8, in Proceedings of the 10th International Conference on Accelerator and Large Experimental Physics Control Systems, Kobe, Japan, 2009 (JACoW, Kobe, Japan, 2009), pp. 659-661, THB006.

[17] T. Nakamura, Bucket-by-bucket on/off-axis injection with variable field fast kicker, in Proceedings of the 2nd International Particle Accelerator Conference, San Sebastián, Spain (EPS-AG, Spain, 2011), pp. 1230-1232 TUPC095.

[18] H. Aoyagi, S. Takahashi, and H. Kitamura, Unipolar signal from a synchrotron radiation beam monitor with microstripline, in Proceedings of the 3rd Annual Meeting of Particle Accelerator Society of Japan and the 31th Linear Accelerator Meeting in Japan, Sendai, Japan, 2006 (PASJ, Tokyo, Japan, 2006), p. 159, TO25.

[19] H. Aoyagi, M. Sano, S. Takahashi, and H. Kitamura, Performance of front-end pulse-by-pulse SR beam monitor with microstripline structure, AIP Conf. Proc. 879, 1010 (2007).

[20] H. Aoyagi, S. Takahashi, and H. Kitamura, Pulse-by-pulse $\mathrm{X}$-ray beam monitor equipped with microstripline structure, in Proceedings of the 10th European Workshop on Beam Diagnostics and Instrumentation for Particle Accelerators (DESY, Hamburg, Germany, 2011), MOPD91, p. 260.

[21] H. Aoyagi and S. Takahashi, Design of x-ray beam position monitor equipped with microstripline structure for undulator beam line, in Proceedings of the 12th Annual Meeting of Particle Accelerator Society of Japan, Tsuruga, Japan (PASJ, Tokyo, Japan, 2015), p. 1224, THP085.

[22] H. Aoyagi, Y. Asano, T. Itoga, N. Nariyama, T. Bizen, K. Fukami, T. Aoki, S. Suzuki, M. Yamaga, T. Otake, T. Tanaka, and H. Kitamura, Electron beam halo monitor for a compact x-ray free-electron laser, Phys. Rev. ST Accel. Beams 16, 032802 (2013).

[23] H. Aoyagi, Y. Asano, T. Itoga, N. Nariyama, T. Bizen, T. Tanaka, and H. Kitamura, Pulse-mode measurement of electron beam halo using diamond-based detector, Phys. Rev. ST Accel. Beams 15, 022801 (2012). 